\title{
Normas de desempenho em tarefa de leitura de palavras/ pseudopalavras isoladas (LPI) para crianças de 10 ano a 7ㅇ ano
}

\section{Developmental standards in a Word/ Pseudoword Reading Task for children in Elementary School}

\section{Jerusa Fumagalli de Salles*}

Universidade Federal do Rio Grande do Sul - UFRGS, Porto Alegre, Rio Grande do Sul, Brasil

\section{Luciane da Rosa Piccolo**}

Universidade Federal do Rio Grande do Sul - UFRGS, Porto Alegre, Rio Grande do Sul, Brasil

\section{Renata de Souza Zamo ${ }^{* * *}$}

Universidade Federal do Rio Grande do Sul - UFRGS, Porto Alegre, Rio Grande do Sul, Brasil

\section{Rudineia Toazza****}

Universidade Federal do Rio Grande do Sul - UFRGS, Porto Alegre, Rio Grande do Sul, Brasil

\begin{abstract}
RESUMO
O instrumento de avaliação de leitura de palavras/pseudopalavras isoladas (LPI), de fácil aplicação e baixo custo, avalia a habilidade de leitura oral (reconhecimento de palavras/ pseudopalavras). São estímulos criteriosamente selecionados conforme regularidade, extensão, frequência e lexicalidade, permitindo avaliar a funcionalidade das rotas de leitura (modelos de dupla-rota). Este estudo apresenta a construção das normas de desempenho (escores totais, em palavras regulares, irregulares e pseudopalavras) para crianças do 1 을 ano a $7^{\circ}$ ano do ensino fundamental, divididas por anos completos de escolarização, idade e tipo de escola. Os resultados mostraram que as crianças de séries e idades mais avançadas apresentaram melhor desempenho em LPI do que as de séries iniciais e mais novas. Os estudantes de escolas privadas tiveram melhor desempenho do que os de escolas públicas. O instrumento pode ser utilizado por diversos profissionais, para complementar a avaliação e subsídio para diagnósticos, planejamento de aulas, encaminhamentos e intervenções.

Palavras-chave: Leitura, Escrita, Alfabetização, Neuropsicologia, Linguagem escrita.
\end{abstract}

\section{ABSTRACT}

The single words/pseudowords reading task (LPI) is an easy to use and low cost instrument which assesses the ability of oral reading 
(words/pseudowords recognition). The stimuli were carefully selected controlled by regularity, length, frequency and lexicality, to allow the evaluation of reading routes (dual-route models) functionality. This study presents how the standards of performance (total scores, scores in regular and irregular words, and pseudowords) were built for children from elementary school, divided by years of school completed, age and type of school. The results showed that elder children (higher grades) had better LPI performance than the younger (lower grades). Students from private schools had better performance than children from public schools. The LPI task can be used by several professionals to complement and benefit the reading assessment, diagnosis, teaching planning, referrals and interventions.

Key-words: Reading, Written, Literacy, Neuropsychology, Written language.

\section{I ntrodução}

O processo de leitura proficiente depende da capacidade de reconhecer as palavras automaticamente e de maneira precisa (CARDOSO-MARTINS, 2008), envolvendo também a compreensão do que é lido (TANNOK, 2000). O reconhecimento de palavras pode ocorrer, conforme modelos de Leitura de Dupla Rota (COLTHEART; CURTIS; ATKINS; HALLER, 1993; COLTHEART; RASTLE; PERRY; LANGDON; ZIEGLER, 2001; ELLIS, 1995), através de um processo visual direto (rota lexical) ou por mediação fonológica (rota fonológica). A funcionalidade da rota fonológica pode ser inferida especialmente pela habilidade de ler pseudopalavras, enquanto que a funcionalidade da rota lexical é demonstrada principalmente pelo desempenho na leitura de palavras reais irregulares (SALLES; PARENTE, 2007).

O desempenho em leitura melhora com o aumento da idade/escolaridade da criança tornando-se automático ao longo das séries escolares (ÁVILA; KIDA; CARVALHO; PAOLUCCI, 2009; PICCOLO, 2010; PICCOLO; SALLES, 2013; SALLES; PARENTE, 2002a; SEIGNEURIC; EHRLICH, 2005; SWANSON; HOWELL, 2001). As crianças adquirem maior experiência com a leitura, conhecimento lexical e das estruturas de combinações dos sons linguísticos, aperfeiçoando ambas as rotas de leitura de palavras (SEIGNEURIC; EHRLICH, 2005; SALLES; PARENTE, 2002a).

Em uma recente revisão de literatura sobre os estudos realizados nos últimos vinte anos na área de reconhecimento de palavras, Lúcio e Pinheiro (2011) demonstraram que as crianças falantes do português parecem utilizar preferencialmente a estratégia fonológica no início da aprendizagem da leitura, que vai sendo gradualmente substituída pelo uso da estratégia lexical. As autoras também perceberam que questões metodológicas - como a forma de aplicação da tarefa de leitura, o tipo de análise estatística utilizada e o número de categorias consideradas na classificação de regularidade das palavras - têm 
interferido nos resultados encontrados. Salles e Parente (2002b), por exemplo, verificaram predomínio da rota fonológica em crianças de segunda série enquanto que os alunos de terceira série usavam predominantemente a rota lexical.

A tarefa de leitura de palavras/pseudopalavras isoladas (LPI), objeto deste estudo, construída por Salles (2001) e Salles e Parente (2002a, $b$, 2007), vem sendo utilizada em várias pesquisas e na avaliação clínica de crianças de séries iniciais de escolarização, tendo permitido encontrar diferenças entre os desempenhos das crianças de acordo com série e tipo de escola (SALLES; PARENTE, 2002a, b, 2007; PICCOLO, 2010; PICCOLO; SALLES, 2013). A influência do contexto escolar (escolas públicas e privadas) no desempenho acadêmico, incluindo o de leitura, tem sido demonstrada no Brasil (CASARIN; WONG; PARENTE; SALLES; FONSECA, 2012; CORSO; SPERB; SALLES, 2013), nas avaliações governamentais (INEP, 2006) e em outros países da América Latina (ARDILA; ROSSELLI; MAMUTE; GUAJARDO, 2005; ROSSELLI; MATUTE; ARDILA, 2006). A escola privada parece contribuir de forma mais evidente do que a pública no desempenho acadêmico (ÁVILA; KIDA; CARVALHO; PAOLUCCI, 2009), o que pode ser associado a aspectos qualitativos da escolarização, tais como método de ensino, nível e profundidade de estimulação, qualidade das relações com colegas e professores (GARDINAL; MARTURANO, 2007), entre outros. Relacionado ao tipo de escola, o nível socioeconômico também é considerado um fator importante para o desenvolvimento da habilidade de leitura (ARDILA; ROSSELLI; MAMUTE; GUAJARDO, 2005; CASTILLO; CHILLÓN; JIMÉNEZ-PAVÓN; ESPERANZA-DÍAZ; MORENO; ORTEGA, 2011; VILLASEÑOR; MARTÍN; DÍAZ; ROSSELLI; ARDILA, 2009; PICCOLO; FALCETO; FERNANDES; LEVANDOWSKI; GRASSI-OLIVEIRA; SALLES, 2012).

Outros instrumentos nacionais para avaliação da leitura de palavras (e habilidades relacionadas) vêm sendo utilizados por fonoaudiólogos, psicólogos, pedagogos, psicopedagogos e professores, como o PROLEC - Provas de Avaliação dos Processos de Leitura (CUETOS; RODRÍGUEZ; RUANO, 1996), adaptado por CAPELLINI, OLIVEIRA; CUETOS (2010), O TDE - Teste de Desempenho Escolar (STEIN, 1994), o TCLPP - Teste de Competência de Leitura de Palavras e Pseudopalavras (SEABRA; CAPOVILLA, 2010), O PROHMELE: Provas de Habilidades Metalinguísticas e de Leitura (CUNHA, 2008), o Teste de Desempenho Cognitivo-Linguístico (TDCL) (CAPELLINI; SMYTHE, 2008), adaptado do International Dyslexia Test - IDT (SMYTHE, EVERATT, 2000; SMYTHE, EVERATT; SALTER, 2004), a Bateria de Recepção e Produção da Linguagem Verbal (SCLIAR-CABRAL, 2003) e a Bateria de Avaliação da Linguagem Escrita e seus Distúrbios (BALESC) (GODOY, 2001). Alguns destes apresentam dados normativos para alguns grupos etários/séries (exemplo: o TCLPP 
apresenta normas de acordo com o nível de escolaridade), mas nem todos são baseados em modelos de processamento de leitura e adotam critérios rígidos de seleção dos estímulos (exemplo: TDE não inclui pseudopalavras).

A normatização de um teste permite que os escores produzidos por ele sejam comparados e contextualizados (PASQUALI, 2001), ajustando à realidade do pesquisador/clínico/educador. Apesar de características demográficas serem consideradas na seleção da amostra normativa de um teste (URBINA, 2007), é importante ressaltar que a comparação com o caso avaliado deve ser feita com cautela, considerando os diferentes contextos de avaliação e de procedência das crianças, especialmente quando tratamos de habilidades verbais.

Este estudo apresenta dados normativos de desempenho de crianças do 1 을 ano a 70 ano do ensino fundamental de escolas públicas e particulares do Rio Grande do Sul em tarefa de leitura de palavras/pseudopalavras isoladas - LPI (SALLES, 2001; SALLES; PARENTE, 2002a, b, 2007).

\section{Método}

\subsection{Participantes}

Foram utilizadas duas amostras no estudo, que seguiram diferentes critérios de seleção. As características dos participantes são descritas nas Tabelas 1 (amostra 1) e 2 (amostra 2).

Amostra 1 (rígidos critérios de inclusão): constituída por 419 crianças, com idades entre 6 e 12 anos $(M=8,99$; $D P=1,98)$, $54,4 \%$ meninas, estudantes de 1 을 ano a 70 ano (zero, ou seja, frequentando o primeiro ano escolar, a seis anos de estudo completos) de escolas públicas e privadas de Porto Alegre-RS. Foram incluídas na amostra crianças sem histórico de doenças neurológicas, psiquiátricas, dificuldades auditivas, dificuldades visuais não corrigidas, dificuldades de aprendizagem, repetência escolar, indicadores de alterações comportamentais e de Transtorno de Déficit de Atenção/Hiperatividade (TDAH), avaliado através da Escala Conners Abreviada para professores (Conners Abbreviated Teacher Rating Scale - CATRS-10) (BRITO, 1987), e desempenho igual ou superior ao percentil 25 no teste Matrizes Progressivas Coloridas de Raven - Escala especial (ANGELINI; ALVES; CUSTÓDIO; DUARTE; DUARTE, 1999).

Amostra 2 (sem critérios rígidos de inclusão, mas excluiu repetentes): constituída por 139 participantes, de primeira $(n=31)$, terceira $(n=71)$ e quarta $(n=37)$ séries do Ensino Fundamental (zero, ou seja, frequentando a primeira série, a três anos completos 
Jerusa Fumagalli de Salles, Luciane da Rosa Piccolo,

Renata de Souza Zamo, Rudineia Toazza

Normas de desempenho em tarefa de leitura de palavras/pseudopalavras

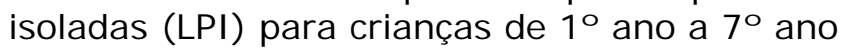

de estudo) de escolas públicas e privadas das cidade de Porto Alegre e Santa Maria ( $n=30$ ), no Rio Grande do Sul, sendo 57,6 \% meninas, com idades entre 6 e 11 anos $(M=8,78, D P=1,33)$.

Tabela 1. Caracterização da Amostra 1 (Frequência ou Média/DP) Quanto à I dade em Anos, aos Anos Completos de Estudo (e ano escolar), ao Sexo, ao Tipo de Escola, ao Nível Socioeconômico (ABEP), ao Percentil no Teste Raven e ao Escore na Escala Conners Abreviada para professores.

\begin{tabular}{|c|c|c|c|c|c|c|c|c|c|c|c|c|c|}
\hline \multirow{3}{*}{$\begin{array}{l}\text { Variável } \\
\text { Tipo de } \\
\text { escola }\end{array}$} & & \multicolumn{12}{|c|}{ Escolaridade em anos completos de estudo/ano escolar } \\
\hline & & \multicolumn{2}{|c|}{ Zero ( 1 o ano) } & \multicolumn{2}{|c|}{$1(2 \mathrm{o}$ ano $)$} & \multicolumn{2}{|c|}{$2\left(3^{\circ}\right.$ ano) } & \multicolumn{2}{|c|}{$3(40$ ano) } & \multicolumn{2}{|c|}{$4(50$ ano) } & \multicolumn{2}{|c|}{5 e $6(6 \% / 70$ ano $)$} \\
\hline & & Pub & Priv & Pub & Priv & Pub & Priv & Pub & Priv & Pub & Priv & Pub & Priv \\
\hline \multirow[t]{2}{*}{$\operatorname{Sexo}(F)$} & Fem & 16 & 17 & 18 & 20 & 17 & 15 & 22 & 19 & 15 & 19 & 30 & 20 \\
\hline & $\mathrm{N}$ & 29 & 28 & 35 & 37 & 32 & 32 & 37 & 34 & 25 & 35 & 55 & 40 \\
\hline Faixa & 6 & 29 & 28 & - & - & - & - & - & - & - & - & - & - \\
\hline etária & 7 & - & - & 35 & 37 & - & - & - & - & - & - & - & - \\
\hline \multirow[t]{9}{*}{ (anos) } & 8 & - & - & - & - & 32 & 32 & - & - & - & - & - & - \\
\hline & 9 & - & - & - & - & - & - & 37 & 34 & - & - & - & - \\
\hline & 10 & - & - & - & - & - & - & - & - & 25 & 35 & - & - \\
\hline & $11 \mathrm{a}$ & - & - & - & - & - & - & - & - & - & - & 55 & 40 \\
\hline & 12 & & & & & & & & & & & & \\
\hline & M & 6,1 & 6,4 & 7,2 & 6,9 & 8,3 & 8,2 & 9,4 & 9,2 & 10,4 & 10,1 & 11,6 & 11,6 \\
\hline & DP & 0,3 & 0,5 & 0,4 & 0,8 & 0,4 & 0,7 & 0,5 & 0,7 & 0,5 & 0,7 & 0,5 & 0,5 \\
\hline & Mín & 6 & 6 & 7 & 6 & 8 & 7 & 9 & 8 & 10 & 9 & 11 & 11 \\
\hline & Máx & 7 & 7 & 8 & 8 & 9 & 9 & 10 & 10 & 11 & 11 & 12 & 12 \\
\hline Classe & A & 3 & 14 & 0 & 18 & 1 & 20 & 1 & 19 & 2 & 17 & 6 & 23 \\
\hline social & B & 15 & 14 & 22 & 19 & 18 & 9 & 15 & 12 & 19 & 14 & 25 & 17 \\
\hline \multirow[t]{2}{*}{$(F)$} & C & 11 & 0 & 12 & 0 & 11 & 3 & 18 & 1 & 4 & 3 & 13 & 0 \\
\hline & D & 0 & 0 & 0 & 0 & 0 & 0 & 1 & 0 & 0 & 0 & 0 & 0 \\
\hline Raven & $M$ & 81,5 & 86,5 & 80,4 & 88,9 & 78,6 & 87,2 & 76,5 & 83,8 & 72,8 & 80,3 & 80,8 & 71,9 \\
\hline (percentil) & DP & 15,7 & 16,6 & 17,7 & 11,1 & 18,6 & 14,9 & 14,7 & 17,8 & 16,0 & 17,6 & 18,7 & 26,1 \\
\hline Escore & $M$ & 4,1 & 4,3 & 3,9 & 4,2 & 3,6 & 4,1 & 3,3 & 4,6 & 2 & 2,3 & 2,9 & 3,2 \\
\hline Conners & DP & 3,6 & 5,0 & 5,4 & 5,0 & 3,7 & 4,5 & 4,1 & 6,3 & 2,9 & 4,3 & 4,4 & 4,0 \\
\hline
\end{tabular}

Nota. Pub = escola pública; Priv = escola privada; ABEP = Associação Brasileira de Empresas de Pesquisas; $\mathrm{F}=$ frequência de crianças; Fem = feminino; Mín = mínimo de acertos; Máx = máximo de acertos.

Tabela 2. Caracterização da Amostra 2 (Frequência ou Média/DP) Quanto à Idade em Anos, ao Número de Anos Completos de Estudo (e Ano escolar), ao Sexo e ao Tipo de Escola. 
Escolaridade em anos completos de estudo/ano escolar

\begin{tabular}{|c|c|c|c|c|c|c|c|c|c|c|}
\hline \multirow{2}{*}{$\begin{array}{c}\text { Variável } \\
\text { Tipo de escola }\end{array}$} & & \multicolumn{2}{|c|}{ Zero ( 1 o ano) } & \multicolumn{2}{|c|}{ Dois ( $3^{\circ}$ ano) } & \multicolumn{2}{|c|}{ Três ( 40 ano) } & \multicolumn{2}{|c|}{ Total } & \multirow[b]{2}{*}{ Total } \\
\hline & & Pub & Priv & Pub & Priv & Pub & Priv & Pub & Priv & \\
\hline \multirow[t]{2}{*}{ Sexo $(F)$} & Fem & 5 & 6 & 26 & 21 & 16 & 6 & 47 & 33 & 80 \\
\hline & $\mathrm{N}$ & 13 & 18 & 37 & 34 & 30 & 7 & 80 & 59 & 139 \\
\hline \multirow[t]{4}{*}{ Idade } & $M$ & 7,2 & 6,4 & 9,6 & 8,3 & 9,9 & 10,1 & 9,3 & 7,9 & 8,7 \\
\hline & DP & 0,7 & 0,6 & 0,5 & 0,4 & 0,3 & 0,7 & 1,1 & 1,3 & 1,4 \\
\hline & Mín & 6 & 6 & 9 & 8 & 9 & 9 & 6 & 6 & 6 \\
\hline & Máx & 9 & 7 & 11 & 9 & 10 & 11 & 11 & 11 & 11 \\
\hline
\end{tabular}

Nota. Pub = escola pública; Priv = escola privada; $F=$ frequência de crianças Fem = feminino; Mín = mínimo de acertos; Máx = máximo de acertos.

\subsection{Procedimentos gerais}

Após contato com as escolas e assinatura dos termos de consentimento Livre e esclarecido (TCLE) pelos pais/responsáveis, a Tarefa de Leitura de Palavras/pseudopalavras Isoladas (LPI) foi aplicada em uma sessão individual com duração média de 15 minutos, na escola do participante, após combinação prévia com o professor. O Teste Matrizes Progressivas Coloridas de Raven Escala Especial (ANGELINI; ALVES; CUSTÓDIO; DUARTE; DUARTE, 1999) foi aplicado coletivamente, em pequenos grupos. Os pais e professores recebiam um prazo para responder e devolver os respectivos questionários (escala). O estudo foi aprovado pelo Comitê de Ética em Pesquisa do Instituto de Psicologia da UFRGS, Porto Alegre, Rio Grande do Sul (número 22048). Os participantes que obtiveram desempenho muito abaixo do esperado pela idade/escolaridade foram encaminhados via escola para atendimento especializado.

\subsection{Instrumentos}

Para seleção da amostra 1:

1. Questionário socioeconômico (ABEP, 2009) e de condições de saúde: contém aspectos socioculturais, de saúde e de escolarização preenchidos pelos pais/responsáveis pela criança.

2. Questionário para professores: inclui informações sobre dificuldades de aprendizagem, métodos de ensino, comportamento do 
aluno em sala de aula, existência de algum diagnóstico e uso de medicação do aluno.

3. Escala Conners Abreviada para professores (Conners Abbreviated Teacher Rating Scale - CATRS-10): instrumento de triagem de indicadores de alterações comportamentais e de Transtorno de Déficit de Atenção/Hiperatividade (TDAH), que apresenta 10 situações de desatenção e de hiperatividade que devem ser pontuadas de acordo com a frequência dos comportamentos da criança (de zero, correspondendo a "nunca", a três, "muito frequente"). A pontuação máxima é de 30 pontos e o ponto de corte sugerido para a população brasileira é percentil 90 (BRITO, 1987).

4. Teste Matrizes Progressivas Coloridas de Raven Escala Especial (ANGELINI; ALVES; CUSTÓDIO; DUARTE; DUARTE, 1999): avalia inteligência não verbal. A classificação resulta da soma dos escores transformados em percentis de acordo com o tipo de escola que a criança frequenta.

Para avaliação da leitura:

Tarefa de Leitura de Palavras/Pseudopalavras Isoladas - LPI (SALLES; PARENTE, 2002a, b, 2007): avalia a precisão na leitura oral de palavras (e pseudopalavras) isoladas, que variam em suas características psicolinguísticas de regularidade (estímulos regulares e irregulares), lexicalidade (palavras reais e pseudopalavras), extensão (estímulos curtos e longos) e frequência de ocorrência na língua (palavras frequentes e não frequentes). O teste consiste de 60 estímulos, sendo 20 de cada categoria (palavras regulares, irregulares e pseudopalavras). Os estímulos são apresentados individualmente, em fonte arial preta, tamanho 24, sobre fundo branco. As palavras reais variam quanto à frequência, segundo a lista de frequência de ocorrência de palavras expostas a crianças na faixa de pré-escola e séries iniciais, desenvolvida por Pinheiro (1996). Foram consideradas palavras curtas, estímulos dissilábicos, constituídos por até cinco letras, e palavras longas, estímulos polissilábicos, contendo oito ou mais letras.

A tarefa é precedida por seis itens-treino, cujo desempenho não é computado. Inicialmente apresenta-se a lista com as 40 palavras reais ( 20 regulares e 20 irregulares) e depois as 20 pseudopalavras, também precedidas de dois itens treino (Apêndice 1). A instrução é que os participantes leiam em voz alta todos os estímulos, logo após sua apresentação. As respostas são gravadas para posterior transcrição.

Para a pontuação, são somados os acertos totais ( máximo $=60$ pontos) e nas categorias palavras reais regulares ( máximo $=20$ pontos) e irregulares (máximo $=20$ pontos) e pseudopalavras ( máximo $=20$ pontos). São computados apenas os pontos dos 
estímulos do teste e não do treino. Na análise quantitativa são considerados acertos as autocorreções imediatas. Este estudo não considerou a análise qualitativa dos tipos de erros, na qual a primeira resposta também é analisada (independente de autocorreção). Mais informações sobre esta análise são encontradas em Salles e Parente (2002a, b, 2007). Na leitura de pseudopalavras são aceitas como corretas todas as possibilidades de leitura conforme regras do Português brasileiro (exemplo: nefochoza, nefoksoza ou nefossoza; erekêla, erequéla ou erecuela).

\subsection{Análise dos dados}

As crianças frequentando $01^{\circ}$ ano do Ensino Fundamental foram consideradas como com "zero anos completos de estudo", frequentando o $2^{\circ}$ ano, com um ano completo de estudo, e assim sucessivamente. Dependendo da amostra, os escores das crianças na LPI foram comparados seguindo os fatores: idade, anos completos de estudo formal, sem considerar repetências, e tipo de escola (públicas e privadas). Para as análises consideraram-se os escores totais e em cada categoria de estímulos (reais regulares, reais irregulares e pseudopalavras) da tarefa de LPI. Na amostra 1, foi utilizado o teste Kruskal-Wallis para comparar o desempenho na tarefa de LPI por anos completo de estudo (escolaridade), idade e tipo de escola. $\mathrm{Na}$ amostra 2, foi realizado o teste Kruskal-Wallis para comparar o desempenho das crianças por séries e idades. Ainda, foram realizados testes de comparação de médias para amostras não-paramétricas (Mann-Whitney) para comparar o desempenho das crianças por tipo de escola apenas para a $3^{a}$ série da amostra 2 e para comparar os desempenhos na tarefa de LPI quanto ao sexo em ambas amostras. Os dados foram analisados através do programa SPSS, versão 19.

Em função dos dados de desempenho das crianças na tarefa de LPI não seguir uma distribuição normal (mas assimétrica), no cálculo dos valores normativos (e pontos de corte) foram utilizados percentis (porcentagem de escores/casos que atingiram determinado escore no teste). Relacionando estes aos escores padronizados (z), o percentil 16 pode ser comparado a - 1 desvio padrão (sugestivo de alerta para déficit); o percentil 7 equivale a $-1,5$ desvio padrão (sugestivo de déficit) e o percentil 2,5 é equivalente a -2 desvios padrão (escores $z$ entre -1,6 e -2,0 são sugestivos de déficit moderado a severo) ${ }^{\mathrm{ii}}$ (STRAUSS; SHERMAN; SPREEN, 2006; FONSECA, SALLES; PARENTE, 2009).

\section{Resultados}

Amostra 1 (critérios de inclusão rígidos) 
As crianças mais escolarizadas tiveram desempenho superior na LPI ao das crianças com menos anos de escolarização, em todas as categorias de LPI: total $\left(x^{2}=197,5 ; p<0,01\right)$, regulares $\left(x^{2}=182\right.$; $p<0,01)$, irregulares $\left(x^{2}=193 ; p<0,01\right)$ e pseudopalavras $\left(x^{2}=\right.$ $109,4 ; p<0,01)$. Da mesma forma, as crianças mais velhas apresentaram desempenho superior em todas as categorias de LPI: total $\left(x^{2}=201,4 ; p<0,01\right)$, regulares $\left(x^{2}=186,7 ; p<0,01\right)$, irregulares $\left(X^{2}=190 ; p<0,01\right)$ e pseudopalavras $\left(X^{2}=109,3 ; p<\right.$ 0,01 ). Observou-se também que os estudantes de escolas particulares tiveram desempenho superior ao de crianças de escolas públicas, em todas as categorias de LPI (teste de Mann-Whitney): total $(U=17122 ; z=-3,12 ; p<0,05)$, regulares $(U=17820,5 ; z=-$ $2,88 ; p<0,05)$, irregulares $(U=17280 ; z=-3,15 ; p<0,05)$ e pseudopalavras $(U=17126 ; z=-3,15 ; p<0,05)$.

Com base em tais resultados, as normas de desempenho na tarefa LPI, para a amostra 1 , são apresentadas considerando-se as variáveis idade ( 6 a 12 anos) e tipo de escola (pública ou particular), na Tabela 3 , e anos completos de estudo ( 0 a 6 , equivalendo a 1 o ano a 7 o ano) e tipo de escola (pública ou particular), na Tabela 4.

Tabela 3. Escore de acertos (total e cada categoria de estímulos) na tarefa de LPI e percentis por idade e por tipo de escola para crianças da amostra $1(n=419)$. 
Jerusa Fumagalli de Salles, Luciane da Rosa Piccolo,

Renata de Souza Zamo, Rudineia Toazza

Normas de desempenho em tarefa de leitura de palavras/pseudopalavras

isoladas (LPI) para crianças de 1 을 ano a 70 ano

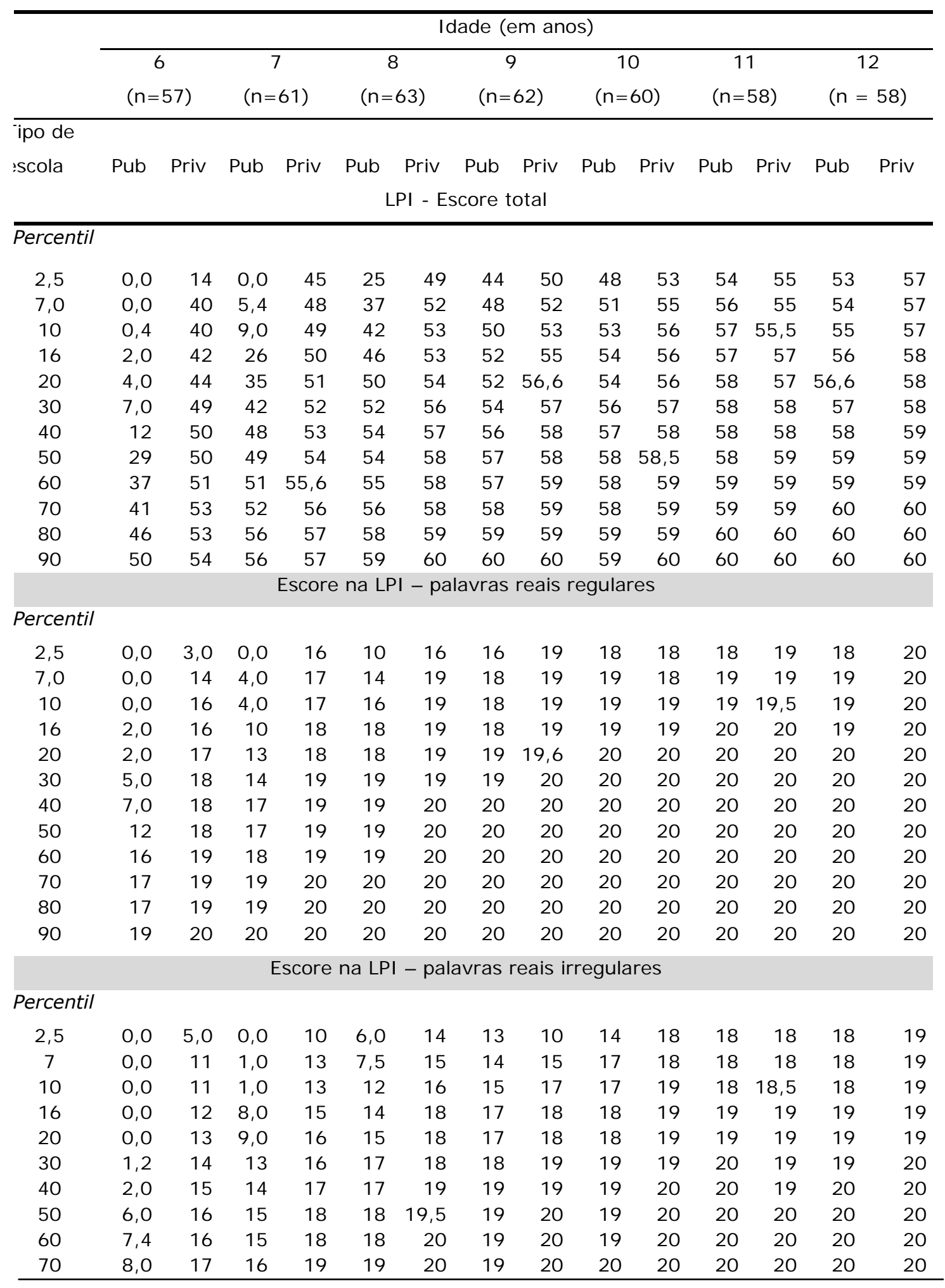

Tabela 4. Escore de acertos (total e em cada categoria de estímulos) na tarefa de LPI e percentis por anos completos de estudo e tipo de escola para crianças da amostra $1(n=419)$. 
Jerusa Fumagalli de Salles, Luciane da Rosa Piccolo, Renata de Souza Zamo, Rudineia Toazza Normas de desempenho em tarefa de leitura de palavras/pseudopalavras isoladas (LPI) para crianças de 1 으o a 70 ano

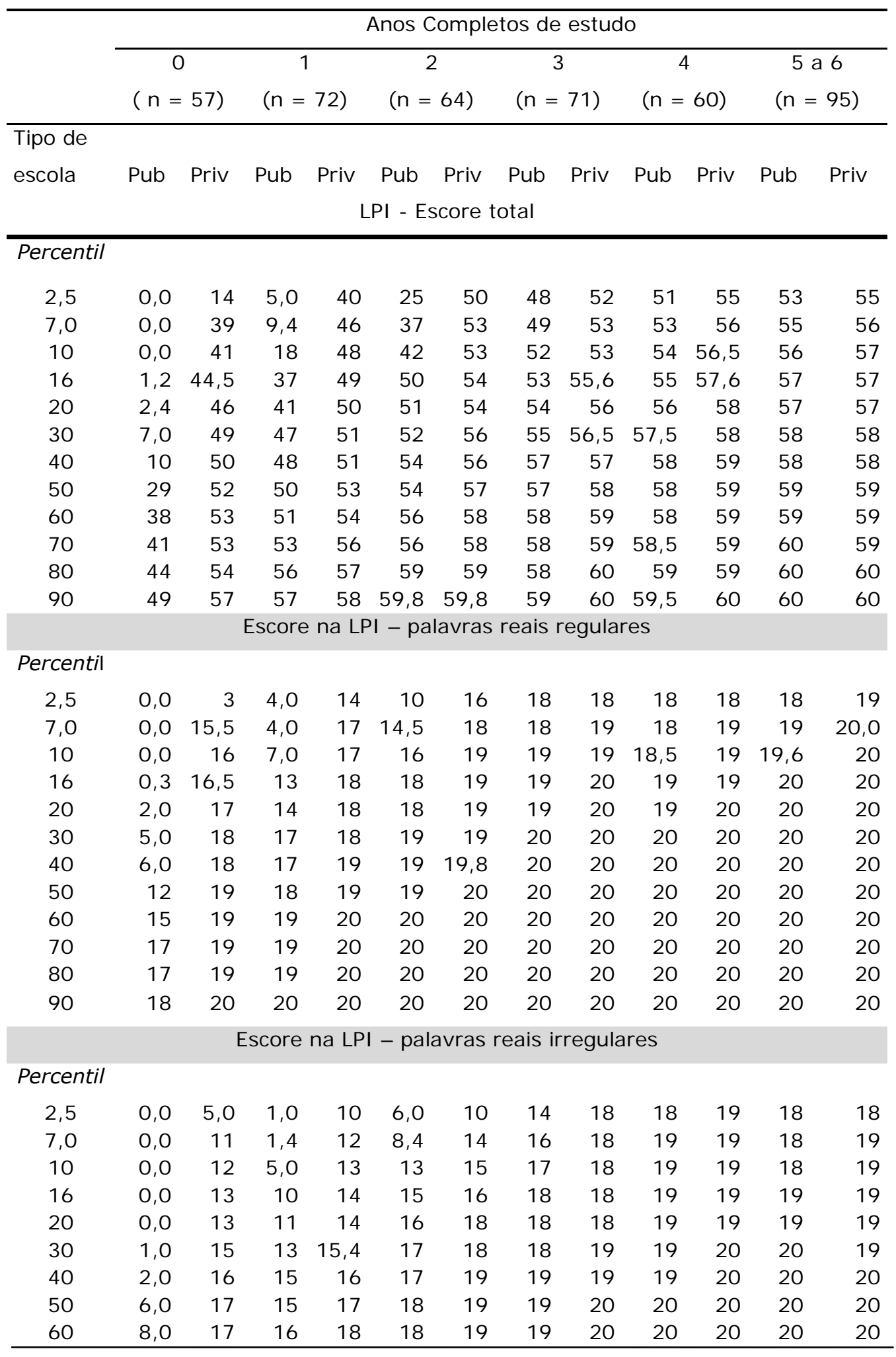

Amostra 2 (critério de exclusão apenas repetência escolar)

Dados normativos na LPI da amostra 2 são apresentados na Tabela 5, por idades, e na Tabela 6, por anos completos de estudo (série 
escolar) e por tipo de escola (para o 3o ano). O número de participantes nesta amostra não é suficiente para apresentar dados normativos por tipo de escola para os demais anos escolares ou por idade. O teste Mann-Whitney mostrou diferenças entre desempenho das crianças por tipo de escola (no 30 ano), sendo que as de escola particular apresentaram desempenho superior às de escola pública em leitura de palavras reais $(U=1840 ; z=-2,24 ; p<0,05)$, tanto regulares $(U=1810 ; z=-2,43 ; p<0,05)$ quanto irregulares $(U=$ 1898; $\mathrm{z}=-1,82 ; \mathrm{p}<0,05)$. O teste Kruskal-Wallis mostrou diferenças de desempenho para todas as idades (com vantagem para as mais velhas) em todas as categorias de LPI: total $\left(X^{2}=39,53\right.$; $p<$ $0,01)$, regulares $\left(x^{2}=34,8 ; p<0,01\right)$, irregulares $\left(x^{2}=49,64 ; p<\right.$ $0,01)$ e pseudopalavras $\left(x^{2}=13,5 ; p<0,01\right)$. A mesma análise mostrou diferenças de desempenho para todos os anos escolares (com vantagem para as mais escolarizadas) em todas as categorias de LPI: total $\left(X^{2}=55,85 ; p<0,01\right)$, regulares $\left(X^{2}=48,05 ; p<\right.$ $0,01)$, irregulares $\left(x^{2}=62,18 ; p<0,01\right)$ e pseudopalavras $\left(x^{2}=\right.$ $19,41 ; p<0,01)$.

Por fim, não houve diferenças de desempenho na tarefa de LPI entre meninos e meninas em nenhuma das amostras estudadas, indicando que as normas servirão para crianças de ambos os sexos.

Tabela 5. Escore de acertos (total e cada categoria de estímulos) na tarefa de LPI e percentis por idade para crianças da amostra $2(n=139)$. 
Jerusa Fumagalli de Salles, Luciane da Rosa Piccolo, Renata de Souza Zamo, Rudineia Toazza Normas de desempenho em tarefa de leitura de palavras/pseudopalavras isoladas ( $\mathrm{LPI}$ ) para crianças de 10 ano a $7 \stackrel{0}{0}$ ano

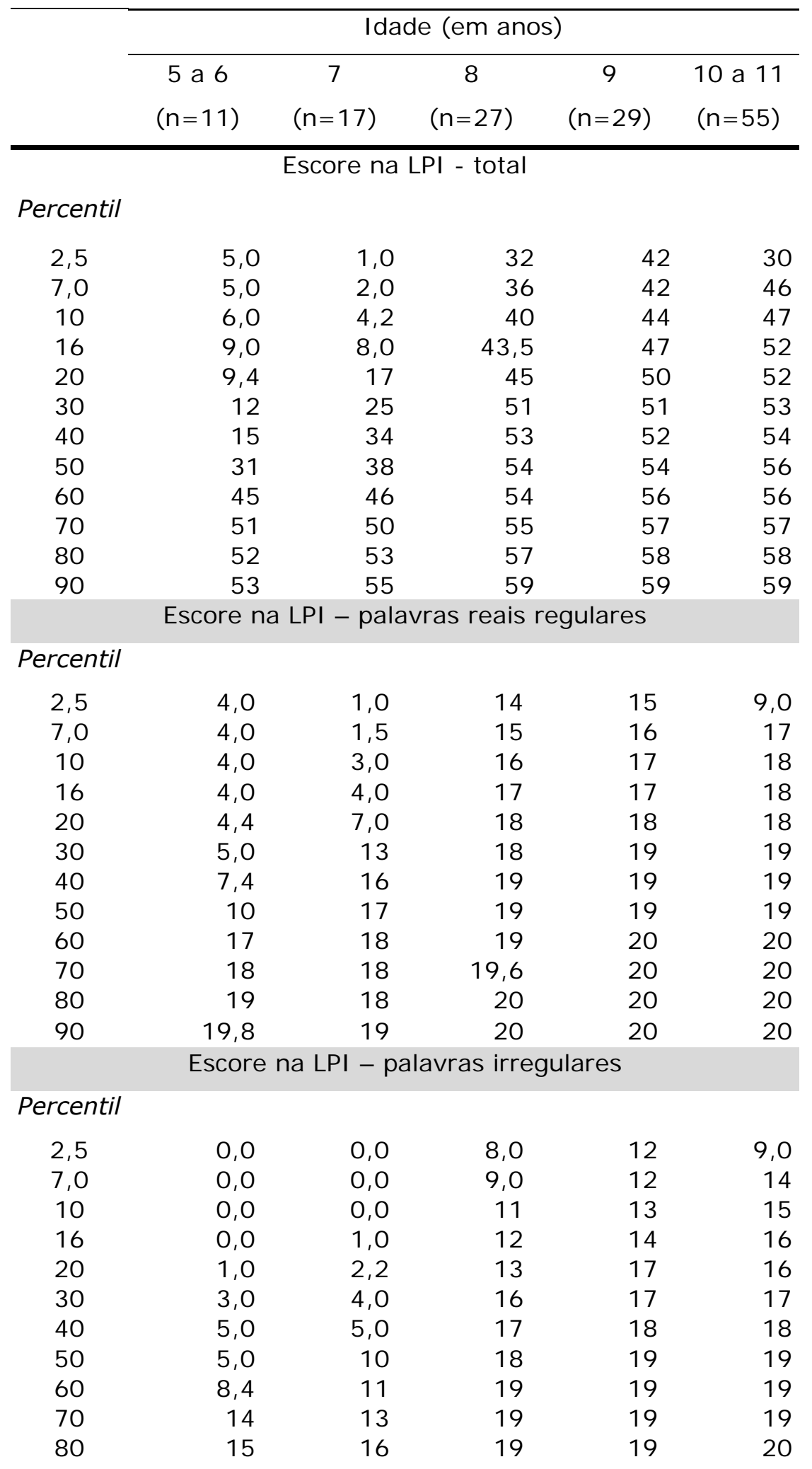

Tabela 6. Escore de acertos (total e cada categoria de estímulos) na tarefa de LPI e percentis por anos completos de estudo (série escolar) e tipo de escola (para 3 a série) para crianças da amostra $2(n=139)$. 
Jerusa Fumagalli de Salles, Luciane da Rosa Piccolo,

Renata de Souza Zamo, Rudineia Toazza

Normas de desempenho em tarefa de leitura de palavras/pseudopalavras isoladas ( $\mathrm{LPI}$ ) para crianças de 10 ano a $7 \stackrel{0}{0}$ ano

\begin{tabular}{|c|c|c|c|c|}
\hline & \multicolumn{4}{|c|}{ Anos completos de estudo (ano escolar) } \\
\hline & \multirow{3}{*}{$\begin{array}{l}\text { Zero (1ㅇ) } \\
(\mathrm{n}=31)\end{array}$} & \multirow{2}{*}{\multicolumn{2}{|c|}{$\begin{array}{l}\text { Dois (3ㅇ) } \\
(n=71)\end{array}$}} & \multirow{3}{*}{$\begin{array}{l}\text { Três (4ㅇ) } \\
(\mathrm{n}=37)\end{array}$} \\
\hline & & & & \\
\hline & & \multicolumn{2}{|c|}{ Publica Privada } & \\
\hline \multicolumn{5}{|c|}{ Escore LPI - total } \\
\hline \multicolumn{5}{|c|}{ Percentil } \\
\hline 2,5 & 1,0 & 23 & 36 & 48 \\
\hline 7,0 & 5,0 & 42 & 41,5 & 52 \\
\hline 10 & 6,0 & 44 & 42,5 & 53 \\
\hline 16 & 9,1 & 46 & 45 & 54 \\
\hline 20 & 11 & 47 & 46 & 55 \\
\hline 30 & 24 & 50 & 51 & 56 \\
\hline 40 & 32 & 52 & 53 & 56 \\
\hline 50 & 38 & 53 & 54 & 57 \\
\hline 60 & 47 & 53 & 55 & 58 \\
\hline 70 & 51 & 55 & 56 & 58 \\
\hline 80 & 52 & 56 & 57 & 59 \\
\hline 90 & 55 & 58 & 59 & 59 \\
\hline \multicolumn{5}{|c|}{ Escore na LPI - palavras reais regulares } \\
\hline \multicolumn{5}{|c|}{ Percentil } \\
\hline 2,5 & 1,0 & 6,0 & 15 & 16 \\
\hline 7,0 & 3,2 & 15 & 15,5 & 18 \\
\hline 10 & 4,0 & 17 & 16,5 & 19 \\
\hline 16 & 4,1 & 17 & 17 & 10 \\
\hline 20 & 5,0 & 18 & 18 & 19 \\
\hline 30 & 10 & 18 & 18 & 20 \\
\hline 40 & 14 & 19 & 19 & 20 \\
\hline 50 & 17 & 19 & 19 & 20 \\
\hline 60 & 18 & 19 & 19 & 20 \\
\hline 70 & 18 & 19 & 20 & 20 \\
\hline 80 & 19 & 20 & 20 & 20 \\
\hline 90 & 19 & 20 & 20 & 20 \\
\hline \multicolumn{5}{|c|}{ Escore na LPI - palavras irregulares } \\
\hline \multicolumn{5}{|c|}{ Percentil } \\
\hline 2,5 & 0,0 & 7,0 & 8,0 & 16 \\
\hline 7,0 & 0,0 & 12 & 10 & 17 \\
\hline 10 & 0,0 & 13 & 12 & 17 \\
\hline 16 & 1,1 & 14 & 12 & 19 \\
\hline 20 & 2,4 & 15 & 14 & 19 \\
\hline 30 & 4,0 & 16 & 17 & 19 \\
\hline 40 & 5,0 & 17 & 17 & 19 \\
\hline 50 & 10 & 17 & 18,5 & 19 \\
\hline 60 & 11 & 18 & 19 & 19 \\
\hline 70 & 14 & 19 & 19 & 20 \\
\hline
\end{tabular}

\section{Discussão}

Estud. pesqui. psicol., Rio de Janeiro, v. 13, n. 2, p. 397-419, 2013. 
Este estudo apresenta dados normativos de desempenho de crianças em idade escolar em um instrumento de fácil aplicação e baixo custo para avaliar a habilidade de leitura em nível de reconhecimento de palavras (e pseudopalavras). Este instrumento pode ser útil para profissionais de várias áreas que trabalham com crianças, como professores, psicólogos, fonoaudiólogos, psicopedagogos, neuropediatras, entre outros profissionais.

As tabelas de dados, dependendo da amostra, foram divididas conforme tipo de escola (pública ou privada), escolaridade (anos completos de estudo formal) e à idade, em função das diferenças de desempenho encontradas nas análises estatísticas. De acordo com o que já havia sido observado no estudo de Salles e colaboradores, que utilizou o mesmo instrumento, há diferenças de desempenho por série/anos de estudo formal, tanto na escola privada (SALLES; PARENTE, 2002b), quanto na pública (CORSO; SPERB; SALLES, 2013; PICCOLO; SALLES, 2013). Resultado semelhante foi encontrado por Capovilla, Varanda e Capovilla (2006), utilizando o Teste de Competência de Leitura de Palavras e Pseudopalavras. Conforme comentado, é esperada progressão das crianças no desempenho em leitura (maior precisão e automaticidade) ao longo das séries escolares e com o aumento da idade (SALLES; PARENTE, 2002a; SEIGNEURIC; EHRLICH, 2005; SWANSON; HOWELL, 2001), pela maior experiência com linguagem (SEIGNEURIC; EHRLICH, 2005; SALLES; PARENTE, 2002a) e aspectos de desenvolvimento da criança. Escolaridade e idade, portanto, são variáveis interrelacionadas e que conjuntamente contribuem para 0 desenvolvimento da leitura.

Em relação ao tipo de escola, os dados deste estudo corroboram os anteriormente encontrados. Por exemplo, utilizando o mesmo instrumento, as médias de desempenho das crianças de 3 a série (escola pública) de Piccolo (2010; PICCOLO; SALLES, 2013) foram mais baixas do que as de 3 a série de escola privada do estudo de Salles e Parente (2002b). Nesse estudo de 2002, a amostra de $2^{a}$ série de escola privada também mostrou desempenhos mais altos na tarefa do que as crianças de 2 a série de escolas públicas estaduais avaliadas por Salles e Parente (2007) e Corso, Sperb e Salles (2013). Estas diferenças tem sido também encontradas em outros trabalhos internacionais, como de Castillo, Chillón, Jiménez-Pavón, EsperanzaDíaz, Moreno e Ortega (2011), realizado com adolescentes espanhóis, no qual fatores socioeconômicos da família (escolaridade e ocupação dos pais) e tipo de escola foram associados ao desempenho cognitivo-linguístico. Além do desempenho acadêmico, a literatura tem indicado que crianças que estudam em escolas privadas apresentam melhor desempenho em avaliações de linguagem, memória e funções executivas (ARDILA; ROSSELLI; MAMUTE; GUAJARDO, 2005; CASTILLO; CHILLÓN; JIMÉNEZ-PAVÓN; 
ESPERANZA-DÍAZ; MORENO; ORTEGA, 2011; VILLASEÑOR; MARTÍN; DÍAZ; ROSSELLI; ARDILA, 2009).

A variável tipo de escola é complexa e pode estar relacionada a diversos fatores. Entre outros, pode-se citar nível socioeconômico, diferenças na oferta de materiais e dedicação dos pais ao aprendizado do filho, método de ensino utilizado pela escola, quantidade e qualidade de estimulação, além de aspectos relacionais da criança com pais, colegas e professores (GARDINAL; MARTURANO, 2007; MARTURANO, 1999).

O fato de o instrumento demonstrar estas mudanças de desempenho conforme critérios de escolaridade, idade e tipo de escola sugere evidências de validade, especialmente nos anos iniciais de escolarização. Em todas as faixas de escolaridade o percentil sete (equivalente ao escore $z$ de menos $1,5 \mathrm{DP}$, sugestivo de déficit) mostrou valores diferentes e menores que o escore teto na tarefa (igual a 60). No entanto, foi observado que nas crianças mais escolarizadas, houve pouca variação dos escores normativos, na amostra 1, a partir do percentil 70.

Em relação aos resultados de escores de acertos nos estímulos regulares, irregulares e nas pseudopalavras, podem ser calculados os efeitos de regularidade (número ou porcentagem de acertos na leitura de palavras regulares menos a porcentagem de acertos na leitura de palavras irregulares) e de lexicalidade (número ou porcentagem de acertos na leitura de palavras reais - frequentes e não frequentes - menos a porcentagem de acertos na leitura de pseudopalavras) (SALLES; PARENTE, 2007). O uso predominante da rota fonológica pode ser inferido pelos altos efeitos de regularidade e da rota lexical pelos altos efeitos de lexicalidade (SALLES; PARENTE, 2007).

Neste estudo, nota-se que as crianças aprimoram o uso de ambas as rotas de leitura (lexical e fonológica) com o aumento da idade e da escolaridade. Consoante à literatura (LÚCIO; PINHEIRO, 2011), as crianças mais novas parecem utilizar preferencialmente a rota fonológica e conforme ficam mais velhas tornam-se mais proficientes em ambas as rotas de leitura. Tal fato pode ser observado nas crianças de menor idade ou escolaridade, pois essas têm escores de desempenho menores em palavras reais irregulares do que em pseudopalavras. Por outro lado, as crianças mais velhas e mais escolarizadas acertam mais palavras irregulares do que as mais novas, pois já aperfeiçoaram o uso da rota lexical. Portanto, o instrumento LPI possibilita a avaliação da rota de leitura preferencial utilizada pela criança conforme sua idade e escolaridade, e fornece normas para as diferentes categorias de palavras/estímulos. Além disso, permite avaliar os tipos de erros e efeitos de regularidade e lexicalidade, conforme mencionado (SALLES; PARENTE, 2007). Dessa forma, contribui com a prática clínica e escolar. 


\section{Considerações finais}

No Brasil, o Instituto Nacional de Estudos e Pesquisas Educacionais Anísio Teixeira - Inep vem produzindo indicadores sobre o sistema educacional brasileiro, apontando problemas graves na eficiência do ensino oferecido pelas redes de escolas brasileiras, como os baixos desempenhos em leitura (Instituto Nacional de Estudos e Pesquisas Anísio Teixeira - INEP, 2012). O Sistema de Avaliação do Rendimento Escolar do Rio Grande do Sul (SAERS) mostrou que 19,5\% das crianças de $2^{\text {a }}$ série/3으 ano da rede estadual de ensino apresentaram desempenho inferior ao nível básico. Esta é uma realidade que necessita de mais atenção, tanto dos órgãos governamentais, quanto dos profissionais e clínicos que trabalham com a faixa de desenvolvimento infantil.

O instrumento em questão possibilita, com a análise quantitativa aqui apresentada, investigar o desempenho (comparativo ao parâmetro de uma amostra normativa), as estratégias de leitura (efeitos psicolinguísticos) e a natureza dos déficits apresentados (em termos de modelo de leitura de dupla-rota), tendo forte base teórica e critérios rígidos de seleção dos itens. Aliado à carência de instrumentos neuropsicológicos brasileiros para crianças e à praticidade e fácil manuseio, aplicação e pontuação, acreditamos que esta tarefa auxilie fornecendo mais subsídios para os processos avaliativos, diagnósticos, planejamento de aulas, intervenções e encaminhamentos. Ainda, a análise qualitativa dos tipos de erros na leitura é uma parte fundamental no processo avaliativo (ver Salles e Parente, 2007).

Salienta-se que o processo de avaliação da leitura (habilidades acadêmicas) precisa ser bastante ampliado, utilizando-se de instrumentos para avaliação da linguagem escrita (receptiva e expressiva), incluindo processos textuais (compreensão de leitura textual e produção escrita), além de outras habilidades neuropsicológicas relacionadas (aritmética, linguagem, memória, atenção, funções executivas). As normas de um instrumento não substituem um clínico/pesquisador com conhecimento e experiência, mas podem ser úteis para serem conjuntamente usadas com outros instrumentos e com dados da história clínica e escolar.

\section{Agradecimentos}

As autoras agradecem ao GNCE (PUCRS), coordenado pela Professora Rochele Paz Fonseca, pelo auxilio na coleta de dados da amostra 1, à 
disponibilidade das escolas que colaboraram com a pesquisa e às famílias que permitiram a participação de seus filhos.

\section{Referências}

ABEP - Associação Brasileira de Empresas de Pesquisa. 2009. Disponível em: <www.abep.org>. Acesso em: 05 nov. 2009.

ANGELINI, A. L.; ALVES, I. C. B.; CUSTÓDIO, E. M.; DUARTE, W. F.; DUARTE, J. L. M. Matrizes progressivas coloridas de Raven: escala especial: manual [Raven's coloured progressive matrices]. São Paulo: CETEPP, 1999.

ARDILA, A.; ROSSELLI, M.; MAMUTE, E.; GUAJARDO, S. The influence of the parent's educational level on the development of executive functions. Development Neuropsychology, Ann Arbor, v.28, 1, p. 539-560, 2005.

ÁVILA, C. R. B.; KIDA, A. S. B.; CARVALHO, C. A. F.; PAOLUCCI, J. F. Tipologia de erros de leitura de escolares brasileiros considerados bons leitores. Pró-Fono Revista de Atualização Científica [online], São Paulo, v. 21, n. 4, p. 320-325, 2009. ISSN 0104-5687. BRITO, G. The Conners Abbreviated Teacher Rating Scale: Development of norms in Brazil. Journal of Abnormal Child Psychology, Atlanta, v. 15, n. 4, p. 511-518, 1987.

CAPELLINI, S. A.; OLIVEIRA, A. M.; CUETOS, F. PROLEC: provas de avaliação dos processos de leitura. São Paulo: Casa do Psicólogo, 2010.

CAPOVILLA, F. C.; VARANDA, C.; CAPOVILLA, A. G. S. Teste de competência de leitura de palavras e pseudopalavras: normatização e validação. PSIC - Revista de Psicologia da Vetor Editora, São Paulo, v. 7, n. 2, p. 47-59, 2006.

CAPELLINI, S. A.; SMYTHE, I. Protocolo de avaliação de habilidades cognitivo-linguísticas: livro do profissional e do professor. Marília, 2008. 100p

CARDOSO-MARTINS, C. Desenvolvimento das Habilidades de Leitura e Escrita. In: FUENTES, D. et al. Neuropsicología: teoria e prática. Porto Alegre: Artmed, 2008. p. 207-229.

CASARIN, F. S.; WONG, C. E. I.; PARENTE, M. A. DE M. P.; SALLES, J. F.; FONSECA, R. P. Comparação de desempenho neuropsicológico entre estudantes de escolas públicas e privadas brasileiras. Spanish J ournal of Psychology, Madrid, v. 15, n. 3, p. 942-951, 2012.

CASTILLO, R. CHILLÓN, P.; JIMÉNEZ-PAVÓN, D.; ESPERANZA-DÍAZ, L.; MORENO, L. A.; ORTEGA, F. B. Associations between parental educational/occupational levels and cognitive performance in Spanish adolescents: The AVENA study. Psicothema, Oviedo, v. 23, n. 3, p. 349-355, 2011. 
COLTHEART, M.; CURTIS, B.; ATKINS, P.; HALLER, M. Models of reading aloud: Dual-rote and parallel-distributed-processing approaches. Psychological Review, Pittsburgh, v. 100, n. 4, p. 589608, 1993.

COLTHEART, M. RASTLE, K.; PERRY, C.; LANGDON, R.; ZIEGLER, J. DRC: A dual route cascaded model of visual word recognition and reading aloud. Psychological Review, Pittsburgh, v. 108, p. 204256, 2001.

CORSO, H. V.; SPERB, T. M; SALLES, J. F. Leitura de palavras e de texto em crianças: efeitos de série e tipo de escola, e dissociações de desempenhos. Letras de Hoje (Impresso), v. 48, p. 81-90, 2013.

CUETOS, F., RODRÍGUEZ, B., RUANO, E. Evaluación de los procesos lectores. Madrid: TEA Ediciones, 1996.

CUNHA, V. L. O. Desempenho de escolares de $1^{a}$ a 4 a séries em provas de habilidades metalinguísticas e de leitura (PROHMELE). 2008. (176f.) Dissertação (Mestrado em Educação) Universidade Estadual Paulista, Marília, 2008.

ELLIS, A. W. Leitura, escrita e dislexia: Uma análise cognitiva. Porto Alegre: Artes Médicas, 1995.

FONSECA, R. P.; SALLES, J. F.; PARENTE, M. A. M. P. Instrumento de avaliação neuropsicológica breve NEUPSILIN. $1^{a}$ ed. São Paulo: Vetor Editora, 2009.

GARDINAL, E. C.; MARTURANO, E. M. Meninos e meninas na Educação Infantil: Associação entre comportamento e desempenho. Psicologia em Estudo, Maringá, v. 12, 3, p. 541-551, 2007.

GODOY, D. M. Testes de consciência fonológica e suas relações com a aprendizagem da leitura no português. 2001. (103f.). Dissertação de Mestrado (Mestrado em Linguística) - Universidade Federal de Santa Catarina, 2001.

Instituto Nacional de Estudos e Pesquisas Anísio Teixeira - INEP. Provinha Brasil: reflexões sobre a prática. Ministério da Educação. 2011. Disponível em: <http://provinhabrasil.inep.gov.br>. Acesso em: 23 fev. 2012.

Instituto Nacional de Estudos e Pesquisas Anísio Teixeira - INEP. Resultados do Enem 2005. Análise do perfil socioeconômico e do desempenho dos participantes. 2006. Disponível em: <http://www. publicacoes. inep.gov.br/detalhes. asp?pub=4054>. Acesso em: 23 fev. 2012.

LÚCIO, P. S.; PINHEIRO, A. M. V. Vinte anos de estudo sobre o reconhecimento de palavras em crianças falantes do português: uma revisão de literatura. Psicologia: Reflexão e Crítica [online]. v. 24, n. 1, p. 170-179, 2011. ISSN 0102-7972.

MARTURANO, E. M. Recursos no Ambiente Familiar e Dificuldades de Aprendizagem na Escola. Psicologia: Teoria e Pesquisa, Brasília, v. 15, 2, p. 135-142, 1999. 
PASQUALI, L. Técnicas de exame psicológico - TEP. São Paulo: Casa do Psicólogo, 2001.

PICCOLO, L. R. Contribuições de variáveis psicossociais e cognitivas e o desempenho em leitura em crianças de uma coorte populacional. 2010. 117f. Dissertação (Mestrado em Psicologia) - Instituto de Psicologia, Universidade Federal do Rio Grande do Sul (UFRGS), Porto Alegre, 2010.

PICCOLO, L. R.; SALLES, J. F. Vocabulário e memória de trabalho predizem desempenho em leitura de crianças. Revista Psicologia: Teoria e Prática, São Paulo, v.15, n.2, p. 180-191, 2013.

PICCOLO, L. R.; FALCETO, O. G.; FERNANDES, C. L.; LEVANDOWSKI, D. C.; GRASSI-OLIVEIRA, R.; SALLES, J. F. Variáveis psicossociais e desempenho em leitura de crianças de baixo nível socioeconômico. Psicologia: Teoria e Pesquisa (UnB. Impresso), v. 28, n.4, p. 389-398, 2012.

PINHEIRO, A. M. V. Contagem de frequência de ocorrência de palavras expostas a crianças na faixa de pré-escola e séries $i$ niciais. Software produzido pela Associação Brasileira de Dislexia ABD, 1996.

ROSSELLI, M.; MATUTE, E.; ARDILA, A. Predictores neuropsicológicos de la lectura en español. Revista de Neurología, Barcelona, v. 42, n. 4, p. 202-210, 2006.

SALLES, J. F. O Uso das rotas de leitura fonológica e lexical em escolares: relação com compreensão, tempo de leitura e consciência fonológica. 2001. 201f. Dissertação (Mestrado em Psicologia do Desenvolvimento) - Instituto de Psicologia, Universidade Federal do Rio Grande do Sul (UFRGS), Porto Alegre, 2001.

SALLES, J. F.; PARENTE, M. A. M. P. Relação entre os processos cognitivos envolvidos na leitura de palavras e as habilidades de consciência fonológica em escolares. Pró-Fono Revista de Atualização Científica, São Paulo, v. 14n. 2, p. 141-286, 2002a.

. Processos cognitivos na leitura de palavras em crianças:

Relações com compreensão e tempo de leitura. Psicologia: Reflexão e Crítica, Porto Alegre, v. 15, n. 2, p. 321-331, 2002b.

. Avaliação da leitura e escrita de palavras em crianças de 2 a série: abordagem neuropsicológica cognitiva. Psicologia: Reflexão e Crítica, Porto Alegre, v. 20, n. 2, p. 218-226, 2007.

SCLIAR-CABRAL, L. Guia prático de alfabetização baseado em princípios do sistema alfabético do português do Brasil. São Paulo: Contexto, 2003.

SEABRA, A.G.; CAPOVILLA, F.C. TCLPP: Teste de competência de leitura de palavras e pseudopalavras (manual e caderno de aplicação). São Paulo: Memnon, 2010.

SEIGNEURIC, A.; EHRLICH, M. F. Contribution of working memory capacity to children's reading comprehension: a longitudinal 
investigation. Reading and Writing, Tygervalley, v. 18, 7, p. 617656, 2005.

Sistema de Avaliação do rendimento escolar do Rio Grande do Sul - SAERS, 2009. Avaliação do rendimento escolar do Rio Grande do Sul. Disponível em: <http://www.saers.caedufjf.net/saers/inicio.faces>. Acesso em: 21, set. 2010.

SMYTHE, I.; EVERATT, J. Dyslexia diagnosis in different languages. In: Peer, L.; G. Reid. Multilingualism, literacy and dyslexia. London, David Fulton Publishers: 2000. p. 12-21.

SMYTHE, I.; EVERATT, J.; SALTER, R. International book of dyslexia: a guide to practice and resources. England: John Wiley \& Sons, LTD, 2004.

STEIN, L. TDE - Teste de desempenho escolar. São Paulo: Casa do Psicólogo, 1994.

STRAUSS, E; SHERMAN, E.M.S; SPREEN, O. Psychometrics Neuropsychological Assessment. In: STRAUSS, E.; SHERMAN E.M.S.; SPREEN, O. A compendium of neuropsychological tests: Administration, norms and commentary. New York: Oxford University Press, 2006. p. 1-43.

SWANSON, H. L.; HOWELL, M. Working memory, short-term memory, and speech rate as predictors of children's reading performance at different ages. Journal of Educational Psychology, Memphis, v. 93, 4, p. 720-734, 2001.

TANNOK, R. Learning Disorders. In: KAPLAN, H.I.; SADOCK, B. J. Comprehensive textbook of psychiatry. $8^{\text {th }}$. ed. Washington: Lippincott Williams \& Wilkins, 2000.

URBINA, S. Fundamentos da testagem psicológica. Porto Alegre: Artmed, 2007.

VILLASEÑOR, E.; MARTÍN, A. L.; DÍAZ, E. G.; ROSSELLI, M.; ARDILA, A. Effects of parents' educational level, school type and gender on the development of attention and memory. Revista Latinoamericana de Psicología, Bogotá, v. 41, n. 2, p. 257-276, 2009.

\section{Endereço para correspondência}

Jerusa Fumagalli de Salles

Instituto de Psicologia, Programa de Pós-Graduação em Psicologia, UFRGS.

Ramiro Barcelos, 2600, sala 114, CEP 90035-003, Porto Alegre, RS - Brasil.

Endereço eletrônico: jerusafs@yahoo.com.br

Luciane da Rosa Piccolo

Instituto de Psicologia, Programa de Pós-Graduação em Psicologia, UFRGS.

Ramiro Barcelos, 2600, sala 114, CEP 90035-003, Porto Alegre, RS - Brasil.

Endereço eletrônico: lucianepiccolo@yahoo.com.br

Renata de Souza Zamo

Instituto de Psicologia, Programa de Pós-Graduação em Psicologia, UFRGS

Ramiro Barcelos, 2600, sala 119, CEP 90035-003, Porto Alegre, RS - Brasil.

Endereço eletrônico: psicozamo@hotmail.com

Rudineia Toazza 
Jerusa Fumagalli de Salles, Luciane da Rosa Piccolo,

Renata de Souza Zamo, Rudineia Toazza

Normas de desempenho em tarefa de leitura de palavras/pseudopalavras

isoladas ( $\mathrm{LPI}$ ) para crianças de 10 ano a $7 \stackrel{0}{0}$ ano

Hospital de Clínicas de Porto Alegre, Ramiro Barcelos, 2350, sala 2202.

CEP 90035-003 Porto Alegre, RS - Brasil.

Endereço eletrônico: rudineiatoazza@yahoo.com.br

Recebido em: 19/03/2012

Reformulado em: 24/04/2013

Aceito para publicação em: 25/04/2013

Acompanhamento do processo editorial: Adriana Benevides Soares

\section{Notas}

*Fonoaudióloga; Doutora em Psicologia do Desenvolvimento - Universidade Federal do Rio Grande do Sul (UFRGS), Profa. Adjunta do Instituto de Psicologia e do Programa de Pós-Graduação em Psicologia da UFRGS. Coordenadora do Núcleo de Estudos em Neuropsicologia Cognitiva (Neurocog), Porto Alegre, RS, Brasil.

** Psicóloga; Mestre e Doutoranda do Programa de Pós-Graduação em Psicologia da UFRGS; Núcleo de Estudos em Neuropsicologia Cognitiva (Neurocog), Porto Alegre, RS, Brasil.

*** Psicóloga; Mestre e Doutoranda do Programa de Pós-Graduação em Psicologia da UFRGS; Núcleo de Estudos em Avaliação Psicológica e Psicopatologia (NEAPP), Porto Alegre, RS, Brasil.

**** Fonoaudióloga; Doutoranda do Programa de Pós-Graduação em Neurociências, Instituto de Ciências Básicas da Saúde (ICBS) da UFRGS e Programa de Transtornos de Ansiedade da Infância e Adolescência (PROTAIA), Hospital de Clínicas de Porto Alegre (HCPA), Porto Alegre, RS, Brasil.

\section{Anexos}


Jerusa Fumagalli de Salles, Luciane da Rosa Piccolo,

Renata de Souza Zamo, Rudineia Toazza

Normas de desempenho em tarefa de leitura de palavras/pseudopalavras

isoladas ( $\mathrm{LPI}$ ) para crianças de 10 ano a $7 \stackrel{0}{0}$ ano

\section{APÊnDICE A - Protocolo de Avaliação da Leitura de Palavras/ pseudopalavras I soladas - LPI (SALLES, 2001; SALLES E PARENTE, 2002a,b, 2007).}

\section{TREI NO:}

\begin{tabular}{|l|l|l|}
\hline Regular & Irregular & Pseudopalavra \\
\hline 1. leão & 4. floresta & 7. naiotise \\
\hline 2. montanha & 5. rosa & 8. lusa \\
\hline 3. bife & 6. maionese & \\
\hline
\end{tabular}

\section{TESTE:}

\begin{tabular}{|c|c|c|}
\hline Regular & Irregular & Pseudopalavra \\
\hline 1. sala & 21. táxi & 41. tapi \\
\hline 2. fada & 22. belo & 42. lobe \\
\hline 3. campo & 23. bosque & 43. cusbe \\
\hline 4. carro & 24. velho & 44. jolha \\
\hline 5. livro & 25. prova & 45. prina \\
\hline 6. operação & 26. alfabeto & 46. beltofa \\
\hline 7. presente & 27. resposta & 47. paresta \\
\hline 8. parágrafo & 28. exercício & 48. azercico \\
\hline 9. importante & 29. transporte & 49. prantorca \\
\hline 10. dinheiro & 30. exemplo & 50. asprona \\
\hline 11. grade & 31. lebre & 51. brele \\
\hline 12. jóia & 32. ônix & 52. unas \\
\hline 13. prata & 33. bloco & 53. clobo \\
\hline 14. surdo & 34. sorte & 54. turse \\
\hline 15. vaga & 35. fixo & 55. cifo \\
\hline 16. orfanato & 36. saxofone & 56. nefoxosa \\
\hline 17. caramujo & 37. aquarela & 57. erequela \\
\hline 18. margarida & 38. crucifixo & 58. crafissoca \\
\hline 19. gelatina & 39. cotonete & 59. tonecote \\
\hline 20. crocodilo & 40. beringela & 60. laberinja \\
\hline
\end{tabular}

\footnotetext{
'Na época desta avaliação vigorava o sistema de séries.

ii Calculo do Escore $z=$ Resultado bruto do participante menos média do grupo normativo ao qual corresponde na variável) dividido pelo desvio-padrão do grupo normativo ao qual corresponde na variável
} 